\title{
A NOVEL DESIGN ON A CD DISC FOR 2-POINT CALIBRATION MEASUREMENT
}

\author{
Marc J. Madou ${ }^{1}$, Yumin Lu' ${ }^{1}$, Siyi Lai ${ }^{2}$, Yije Juang ${ }^{2}$, L. James Lee ${ }^{2}$, Sylvia Daunert ${ }^{3}$ \\ ${ }^{1}$ Department of Materials Science and Engineering, The Ohio State University, Columbus, OH 43210 \\ ${ }^{2}$ Department of Chemical Engineering, The Ohio State University, Columbus, OH 43210 \\ ${ }^{3}$ Department of Chemistry, University of Kentucky, Lexington, KY 50506
}

\begin{abstract}
Today the Si/polymeric medical diagnostic sensors that are on the market only feature a 1-point calibration [1]. Such a measurement results in less accurate sensing and more in-factory sensor rejection. The 2-point calibration fluidic method introduced here will alleviate some of the shortcomings of such current miniature analytical systems. Our fluidic platform is a multipurpose micro analytical laboratory on a compact disc (CD) $[2,3]$. This system is based on the centrifugal force, in which fluidic flow can be controlled by the spinning rate of the CD and thus a whole range of fluidic functions including valving, mixing, metcring, splitting, and separation can be implemented. Furthermore, optical detection such as absorption and fluorescence can be incorporated into the $C D$ control unit to obtain signals from pre-specified positions on the disc.
\end{abstract}

Keywords: microfluidics, centrifuge, SU-8, photolithography.

\section{INTRODUCITON}

The lab CD platform technology we are developing features fluidic structures micromachined into a plastic $\mathrm{CD}$. In the manufacture, we rely on traditional mechanical machining and on lithography based techniques. The centrifugal force due to the rotation of the $C D$ provides the pumping force for the release and flow of reagents and analytes. No external pumps and valves are needed to control the fluids. We used computer numcrical control (CNC) milling to mechanically manufacture the 2-point calibration system on a $\mathrm{CD}$. Although $\mathrm{CNC}$ machining may not achieve satisfactory absolute tolerance, its accessibility and rapid prototyping capability enables us to conduct various investigations concerning microfluidic dynamics faster.

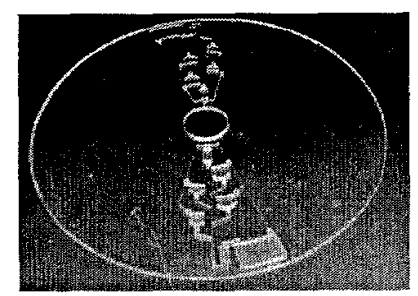

Figure 1. (a) Two 2point calibration units on a $C D$

(b) Functional diagram of a single 2-point calibration unit.
For smaller fluidic features (e.g. channels with $\varnothing<80 \mu \mathrm{m}$ ) and mass production, we are making the CD with a combination of photolithography and replication tools. In this approach, a thick layer of SU-8 (SU-8-100, MicroChem, Inc.) is spin-coated on a metal covered plastic $\mathrm{CD}$, followed by photolithography to pattern the microfluidic structures. After development, nickel is electroplated to make a metal-insert mold which can be used for hot embossing or injection molding.

In our 2-point calibration design (Fig 1), the liquid flows in the order of calibrant 1 , wash1, calibrant 2 , wash 2 , and sample to the optrode chamber by increasing the rotation speed. The same optrode chamber is used for measuring calibrants and sample to eliminate artificial system errors common with devices using separate chambers for measuring sample and calibrants.

For optical detection purposes, chromoionophores are incorporated into small chambers close to the rim of the CD. To demonstrate the functionality of the 2-point calibration system, we deposited a potassium ion selective membrane in the optrode chamber using a micro-delivery set-up (Nano-plotter, from GeSiM) [4]. The membrane is composed of Poly(vinyl chloride) (PVC), valinomycin (ionophore), dioctyl sebacate (plasticizer), potassium tetrakis(4-chlorophenyl) borate and chromoionophore for detection at $640-\mathrm{nm}$ wavelength. In order to identify the proper polymeric material with the lowest protein adsorption and minimal optical interference with the optical measurement, the protein adsorption characteristics and optical absorption properties of seven candidate polymers for the CD platform were evaluated.

\section{THEORY}

\section{Fluid Propulsion}

In order to sequentially valve fluids through a monotonic increase of rotational rate, we designed a structure with progressively higher "burst" frequencies. The burst frequency is the frequency at which the fluidics are released from their reservoirs.

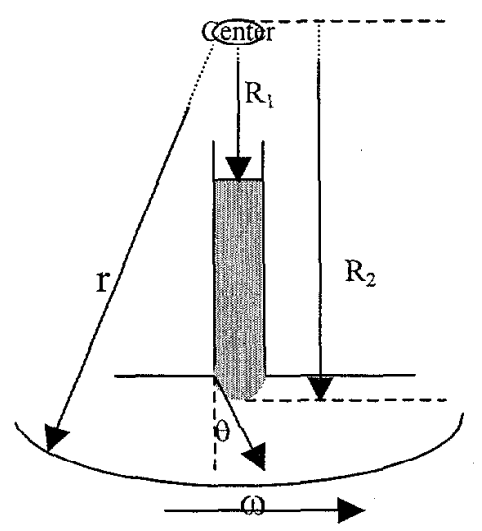

Figure 2. Schematic illustration of fluid propulsion in centrifugal microfluidics. 
In the LabCd platform, the centrifugal force provides the pumping force, while the capillary force at junctions inhibits flow. This constitutes a RPM-dependent capillary "valve" controlling the release and flow of fluids. The two opposing forces can be described as follows (see Figure 2).

The pumping force $\left(P_{c}\right)$ due to centrifugal force is given by:

$$
\frac{d P_{c}}{d r}=\rho \omega^{2} r
$$

Here $\rho$ is the density of the liquid, $\omega$ is the angular velocity of the $\mathrm{CD}$ platform, $r$ is the distance between a liquid element and the center of the $\mathrm{CD}$,

Integration of equation (1) from $r=R_{1}$ to $r=R_{2}$ gives:

$$
\Delta P_{c}=\rho \omega^{2}\left(R_{2}-R_{1}\right) \frac{R_{2}+R_{1}}{2}=\rho \omega^{2} \Delta R \cdot \bar{R}
$$

The capillary force $\left(P_{s}\right)$ due to interface tension is given by:

$$
\Delta P_{s}=\frac{\gamma \cos \theta \cdot C}{A}
$$

Here, $\gamma$ is the interface tension, $\theta$ is the contact angle, $A$ is the cross-section area of the capillary and $\mathrm{C}$ is the associated contact line length.

The liquid will he released when $\Delta \mathrm{P}_{\mathrm{c}}$ is greater than $\Delta \mathrm{P}_{\mathrm{s}}$, consequently the burst frequency calculated from equations 1 and 3 is given by:

$$
f_{b} \geq\left(\frac{\gamma \cos \theta \cdot C}{\pi^{2} \rho \cdot \bar{R} \cdot \Delta R \cdot 4 A}\right)^{\frac{1}{2}}=\left(\frac{\gamma \cos \theta}{\pi^{2} \rho \cdot \bar{R} \cdot \Delta R \cdot d_{H}}\right)^{\frac{1}{2}}
$$

Here $d_{H}$ is so called hydrodynamic diameter of the channel.

\section{2-point calibration}

By appropriately choosing the channel diameter and reservoir dimensions and locations, we designed a 2-point calibration system, which is capable of 5 sequential fluid movement steps. The liquid flows in the order of calibrant 1 , wash 1 , calibrant 2 , wash 2 , and sample to the optrode chamber by increasing the rotating speed.

Table 1 shows the 2-point calibration design parameters for the $\mathrm{CNC}$ machined $\mathrm{CD}$. Figure 3 shows the calculated burst frequencies of the reservoirs in the 2-point calibration platform.

A typical 2-point calibration curve is shown in Figure 4. The slope (sensitivity) is determined hy the measurements of calibrant 1 and calibrant 2 . The ion concentration of the unknown sample is deduced from this calibration curve.

Table 1: Parameters of CNC machined CD platform

\begin{tabular}{|c|l|l|l|l|}
\hline Reservoir & Thickness & Volume & $\begin{array}{l}\text { Burst } \\
\text { RPM }\end{array}$ & $\begin{array}{l}\text { Observed } \\
\text { bust RPM }\end{array}$ \\
\hline Sample & $800 \mu \mathrm{m}$ & $10.47 \mu \mathrm{L}$ & 1222 & 1126 \\
\hline Wash 2 & $800 \mu \mathrm{m}$ & $18.61 \mu \mathrm{L}$ & 1032 & 1026 \\
\hline Calibrant 2 & $800 \mu \mathrm{m}$ & $9.56 \mu \mathrm{L}$ & 832 & 822 \\
\hline Wash 1 & $800 \mu \mathrm{m}$ & $18.03 \mu \mathrm{L}$ & 623 & 622 \\
\hline Calibrant 1 & $800 \mu \mathrm{m}$ & $11.79 \mu \mathrm{L}$ & 427 & 524 \\
\hline Optrode & $500 \mu \mathrm{m}$ & $6.81 \mu \mathrm{L}$ & N/A & N/A \\
\hline Waste & $800 \mu \mathrm{m}$ & $84.35 \mu \mathrm{L}$ & N/A & N/A \\
\hline
\end{tabular}

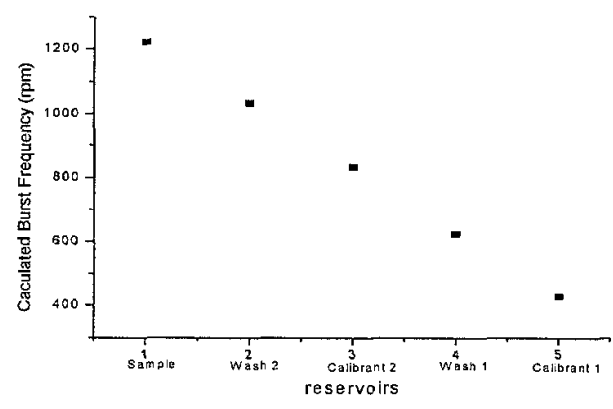

Figure 3. A plot of calculated burst frequencies.

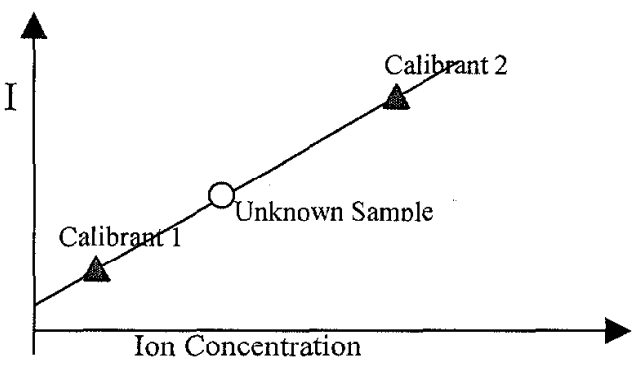

Figure 4. A typical 2-point calibration curve.

\section{EXPERIMENTAL}

\section{Computer Numerical Control (CNC) machined CD}

Numerical control machining is a method of automatically operating a machine. It has been used in industry for over 40 years [5]. CNC machining features an added on-board computer, which enables programmed control of the machine. The typical cutting tools used in CNC machining are: milling, drills, multifacet drills, boring, tapping, angle milling, etc [5]. Two CNC machined 2-point calibration units in a polycarbonate disc are showed in Figure 1.

Although $\mathrm{CNC}$ machining has advantages such as easy accessibility and rapid prototyping capability, it has several limitations in this application. For example, CNC milling can hardly fabricate samples with feature size less than 80 microns or make smooth turns. Another concern is the aspect ratio (the ratio of depth/width). The aspect ratio achieved by $\mathrm{CNC}$ machining is typically not high ( 0.5 in our case).

Considering those limitations of CNC machining, we are exploring alternative manufacturing methodologies. Photolithography with deep-UV resist followed by electroplating and molding is our preferred method for LabCD platform fabrication

\section{SU-8 BASED CD}

Thick photoresist based lithography is widely used in micromachining. It has several advantages, for example high aspect ratio features are available (over 20 reported) [6] and very small feature sizes can be obtained $[7,8]$. Furthermore, it is much easier to fabricate any desired pattern compared to $\mathrm{CNC}$ machining. For example, the shape of the reservoir can be easily changed from square to circular or drop-like shape with lithography. 
We used SU-8, which is a negative resin-based photoresist, in this application. Firstly a thick layer of SU-8 was coated on a metal-covered plastic $\mathrm{CD}$. Metal ( $\mathrm{Ti} / \mathrm{Au})$ is used to protect the CD's surface from the SU-8 developer. To decrease the thermal stress in the SU-8, the sample was soft-baked at $70^{\circ} \mathrm{C}$ for 1 hour and then at $95^{\circ} \mathrm{C}$ for 3 hours. The Exposure step was performed on a PLA-501 FA Canon mask aligner. The post-bake was carried out at $60^{\circ} \mathrm{C}$ for 20 minutes and then at $90^{\circ} \mathrm{C}$ for 30 minutes. Again, this was to minimize the thermal stress.

After development in PMGA SU-8 developer (MicroChem, Inc.), microfluidic structures such as channels and reservoirs shown in Figure 5 resulted. The width of the channel in Figure $5 b$ is $50 \mu \mathrm{m}$ and the height is $200 \mu \mathrm{m}$ (aspect ratio $=4$ ).

The mechanical and optical properties of SU-8 structures need further investigation. However, microfluidic components fabricated in SU-8 may in some instances be directly used in platform evaluation.

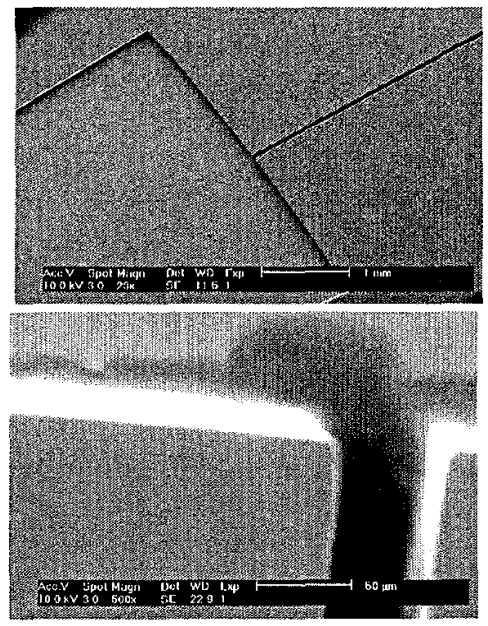

Figure 5(a) (b). SEM of the microchannel and reservoir manufactured by photolithography in SU-8.

\section{PLASTIC REPLICATION}

Firstly, an SU-8 structure is fabricated by photolithography as described in the previous section. The resulting structure features the same pattern as the desired final product. Electroplating is then carried out to replicate a metal mold (typically nickel) from the SU-8 structure. Such mold can be used in hot embossing or injection molding for mass production of the CD platform [9].

Polycarbonate (PC), Optical Quality Polycarbonate (OQPC), Polyurethane (PU), Polydimethyl Siloxane (PDMS), Polystyrene (PS), Polyvinyl butyral (PVB), and Polymethylmethacrylate (PMMA) were used in this application.

In the fabrication of regular PC, OQPC, PMMA and PS discs, the hot embossing process was applied and the experimental setup is shown in Figure 6. The mold was first brought in contact with the polymer substrate. Both of them were heated up above the glass transition temperature ( $\mathrm{Tg}$ ), followed by hot embossing process for a few seconds. After the mold and polymer substrate were cooled down below $\mathrm{Tg}$, the mold was then separated from the polymer substrate with the microstructures fully transferred into it. For rubber-like polymers such as PU and PVB, same procedures were applied except the heating-temperature was $105^{\circ} \mathrm{C}$. High temperature was adopted in order to prevent the rubbery material from springing back after embossing. As to PDMS, a casting method was employed, which is shown in Figure 7. A holding ring was made to hold the resin and the mold. After the resin is poured into the holding ring, it cured slowly and became rubbery PDMS with pattern replicated onto it, which can be easily peeled off from the mold.

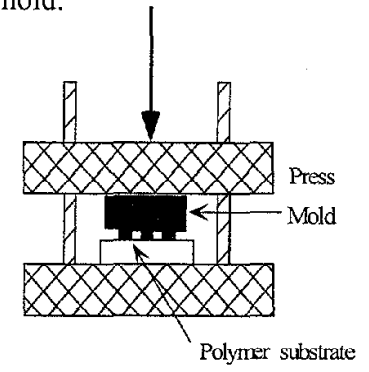

Figure 6. Schematic of hot embossing.

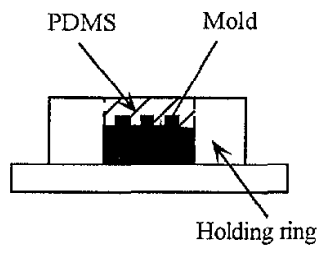

Figure 7. Schematic of casting.

\section{Optical Sensor incorporated CD}

We deposited a potassium ion selective membrane in the optrode chamber for the measurement of the ion concentration in the calibrantas and unknown sample. The deposition is performed by a GeSiM Micro pipetting system (Nano-plotter). Table 2 represents the composition of the membrane.

When the testing solution contacts the membrane, the potassium ion is extracted into the membrane and bound with the valinomycin. To ensure the electrical neutrality within the membrane, the chromoionophore loses a hydrogen ion which goes into the buffered sample solution. The loss of the hydrogen ion causes a change in the optical properties of the chromoionophore (absorption, fluorescence, etc.), which is what we measure. The magnitude of this change depends on the ion concentration of the testing solution. Therefore, we can obtain the ion concentration by measuring the change in the optical properties of the chromoionophore.

Table $2 . \mathrm{K}^{+}$selective membrane composition

\begin{tabular}{|l|l|}
\hline Dioctyl sebacate & $65 \mathrm{mg}$ \\
\hline PVC & $33 \mathrm{mg}$ \\
\hline valinomycin & $2 \mathrm{mg}$ \\
\hline $\begin{array}{l}\text { Chromoionophore } \\
\text { (ETH5350) }\end{array}$ & $\begin{array}{l}50 \mathrm{~mole} \% \text { relative to } \\
\text { the ionophore }\end{array}$ \\
\hline $\begin{array}{l}\text { Potassium tetrakis(4- } \\
\text { chlorophenyl)borate }\end{array}$ & $\begin{array}{l}50 \text { mole\% relative to } \\
\text { the ionophore }\end{array}$ \\
\hline
\end{tabular}

\section{RESULTS AND DISCUSSIONS}

\section{Protein adsorption characteristics of polymer materials for CD platform}

Since the proteins might be used in the CD platform, protein adsorption characteristics of the CD substrate were studied to identify a proper polymeric material for this application. Seven polymeric materials were evaluated. They are: Polycarbonate, OQPC, Polyurethane, PDMS, Polystyrene, PVB, and PMMA.

It was found that $\mathrm{PC}$ and PMMA have comparable protein adsorption characteristics to that of PS. PVB was not suitable for this application since it is too sensitive to protein and as a consequence, its optical properties are greatly affected upon the exposure to the protein.

\section{Burst frequencies of the 2-point calibration platform}

The CNC machined polycarbonate disc was covered with another PC plate and tested for the burst frequencies of the reservoirs. The effect of the cover material's properties (e.g. stiffness, interface tension, etc) on the burst frequency was 
observed. The CD covering is an important issue when testing the burst frequency because the covering material may affect the contact angle and the interface tension between the solution and substrate. When testing the burst frequency of different chambers, two methods of $C D$ covering were tried: soft cover (cover the CD with the Scotch tape) and hard cover (cover the CD with a polycarbonate plate). Since the Scotch tape is flexible, it can be bulged up when pressure is applied, higher burst frequencies than the calculated values were expected and the experimental results confirmed it.

Table 1 shows the test results on a polycarbonate covered $\mathrm{CD}$. Considering the tolerance of $\mathrm{CNC}$ machining and the measuring deviation, the results are quite consistent with the calculated frequencies.

\section{CONCLUSION}

A 2-point calibration measurement platform based on the centrifugal pumping force and RPM-dependant "valves" are developed. Three fabrication methodologies: CNC machining, SU8 photolithography and polymer replication are discussed and contrasted. The burst frequencies of the $C N C$ machined $C D$ were tested and compared to the calculated values. Optical sensor is incorporated into this platform for detection of ion concentration. The protein adsorption characteristics of seven polymeric materials were evaluated. By using the micromachining technology, the fluidic structure's size is decreased to micron scale. Thus the sample volume utilized in the LabCD platform is reduced to micro/naro liter. Multi analysis and high throughput screening can be achieved by different designs in the LabCD.

\section{Acknowledgment}

The authors wish to thank Paul Green for his work in CNC machining, Pang-Hung Hsu, Chee Guan Koh and Harry He for their contributions. This work is supported by NASA.

\section{REFERENCES}

1. Jacobs, E., Vadasdi, E., Sarkozi, L., Coman, N. “ Analytical Evaluation of i-STAT portable Clinical Analyzer and Use by Nonlaboratory Health-Care professionals". Clin. Chem. 1993, 39(6), 1069.

2. Duffy, D. C., Gills, H. L., Lin, Joe, Sheppard, N. F., kellogg, G. "Microfabricated Centrifugal Microfluidic Systems: Characterization and Multiple Enzymatic Assays" J. Anal. Chem. 1999, 71, 4669.

2. Madou, M. J., Kellogg, G. J. "The LabCD" ${ }^{\mathrm{TM}}$ : A centrifugebased microfluidic platform for diagnostics". SPIE. San Jose, CA (1998).

3. GeSiM mbH, Bautzener Landstrasse 45, D-01454 Grosserkmannsdorf Germany.

5. Valentino, J. V., Goldenberg J. "Introduction to computer numerical control" Prentice Hall, 1993.

6. Report on $\mathrm{NANO}^{\mathrm{TM}} \mathrm{SU}-8$ resists for MEMS/microsystem application by MicroChem Corp. Nov, 1999.

7. K. Y. Lee, N. Labianca and S.A. Rishton " Micromaching Application of a High Resolution Ultrathick Photoresist" $J$. of Vacuum Science and Technology B, 13(6): 3012-3016 (1995).

8. H. Lorenz, M. Despont, N. Fahni, N. Labiaca, "SU-8: A lowcost negative resist for MEMS" SUSS report, 10:1-3, Third/Fourth Quarter (1996).

9. H. Schift, R. W. Jaszewski, C. David, and J. Gobrecht "Microelectronic Engineering", 46, 121 (1999). 